\title{
Ruthenium(III) Catalysis in Perborate Oxidation of 5-Oxoacids
}

\author{
S. Shree Devi, ${ }^{1,2}$ P. Krishnamoorthy, ${ }^{3}$ and B. Muthukumaran ${ }^{4}$ \\ ${ }^{1}$ Research and Development Centre, Bharathiar University, Coimbatore 641 046, India \\ ${ }^{2}$ The Department of Chemistry, Arignar Anna Government Arts College, Cheyyar 604 407, India \\ ${ }^{3}$ Department of Chemistry, Dr. Ambedkar Government Arts College, Chennai 600 039, India \\ ${ }^{4}$ Department of Chemistry, Presidency College, Chennai 600005, India
}

Correspondence should be addressed to B. Muthukumaran; dr.muthukumaran@yahoo.com

Received 16 May 2014; Revised 2 August 2014; Accepted 3 August 2014; Published 24 August 2014

Academic Editor: Taicheng An

Copyright (C) 2014 S. Shree Devi et al. This is an open access article distributed under the Creative Commons Attribution License, which permits unrestricted use, distribution, and reproduction in any medium, provided the original work is properly cited.

Ruthenium(III) catalyzes perborate oxidation of substituted 5-oxoacids in acidic solution. The catalyzed oxidation is first order with respect to the oxidant and catalyst. The rate of ruthenium(III) catalyzed oxidation displays the Michaelis-Menten kinetics on the reductant and is independent of $\left[\mathrm{H}^{+}\right]$of the medium. Hydrogen peroxide is the reactive species of perborate and the kinetic results reveal formation of ruthenium(III) peroxo species-5-oxoacid complex. Electron-releasing substituents accelerate the reaction rate and electron-withdrawing substituents retard it. The order of reactivity among the studied 5-oxoacids is $p$-methoxy $\gg p$-methyl $>p$-phenyl $>-\mathrm{H}>p$-chloro $>p$-bromo $>m$-nitro. Activation parameters are evaluated using Arrhenius and Eyring's plots. A mechanism consistent with the observed kinetic data is proposed and discussed. A suitable rate law is derived based on the mechanism.

\section{Introduction}

Sodium perborate $\left(\mathrm{NaBO}_{3} \cdot 4 \mathrm{H}_{2} \mathrm{O}\right)$ is a cheap and environment friendly large-scale industrial chemical used extensively in detergents as a bleaching agent. It is a mild oxidant, and the search for suitable catalysts for perborate oxidation is of interest. It is a convenient source of hydrogen peroxide [1], commercially, industrially, and also in the laboratory. Proton magnetic resonance (PMR) spectral analysis [2] and X-ray diffraction studies [3] conclude perborate as a true peroxysalt with water of crystallisation. It is an effective reagent in organic synthesis, and acetic acid is the solvent of choice [4]. Perborate in aqueous solution yields hydrogen peroxide and the kinetic studies in aqueous and partly aqueous acidic media confirm perborate oxidation as hydrogen peroxide oxidation $[5,6]$. This stable and easily handled crystalline substance oxidizes organic sulphides [7], anilines [8], and indole [9]. This communication reports ruthenium(III) catalysis in perborate oxidation of 5-oxoacids. Vanadium(V) also catalyzes perborate oxidation of 5-oxoacids [10].

In 5-oxoacids and their phenyl substituted compounds, two carbon atoms separate the carbonyl and the carboxyl groups, and so they behave both as oxocompounds and as acids without the direct influence of the other group. In acidic medium, 5-oxoacids undergo enolization, and on selective oxidation, they result in malonic and substituted benzoic acids, which find widespread applications in synthetic organic chemistry. Hence, 5-oxoacids are attractive substrates in terms of their mechanistic aspects. 5-oxocid is an attractive substrate in terms of its enolization. In strong acid medium the substrate undergoes enolization. The reactive species of the substrate is reported in the literature to be the enol form [11, 12]. Studies of the oxidation of various organic compounds by perborate have attracted considerable attention. A thorough literature survey reveals that relatively little work on the oxidation of oxoacids have been reported so far $[13,14]$. Although the perborate oxidations of organic compounds have been studied, there seems to be no report on a systematic kinetic study of the oxidation of 5-oxoacids by perborate, and we report here for the first time the kinetics and mechanism of ruthenium(III) catalysis in perborate oxidation of substituted and unsubstituted 5-oxoacids. The various unsubstituted and substituted 5-oxoacids (S1-S7) employed in the present study are listed in Scheme 1. 


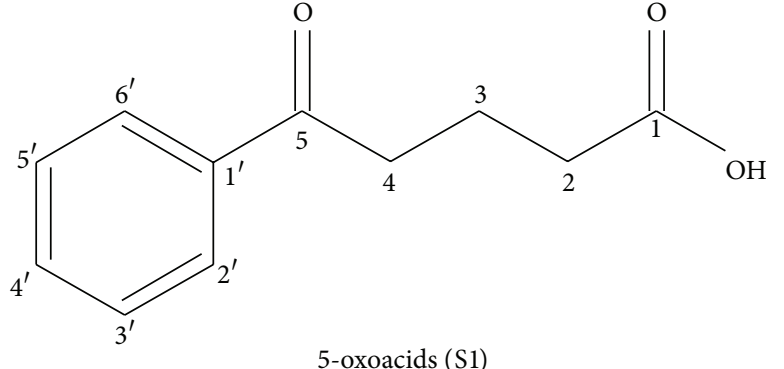

$\begin{array}{ll}\text { S1: Unsubstituted } & \text { S5: } 4^{\prime} \text {-chloro } \\ \text { S2: } 4^{\prime} \text {-methoxy } & \text { S6: } 4^{\prime} \text {-bromo } \\ \text { S3: } 4^{\prime} \text {-methyl } & \text { S7: } 3^{\prime} \text {-nitro } \\ \text { S4: } 4^{\prime} \text {-phenyl } & \end{array}$

SCHEME 1

\section{Experimental}

2.1. Materials. Sodium perborate, $\mathrm{NaBO}_{3} \cdot 4 \mathrm{H}_{2} \mathrm{O}$ (Riedel), and ferrous sulphate (Merck) were used as received. Other chemicals were of AR grade. Analytical grade acetic acid (BDH) was refluxed for $6 \mathrm{~h}$ over chromium(VI) oxide and distilled through a column. Solutions of perborate were prepared freshly and standardized iodometrically. Kinetics of the oxidation in aqueous sulphuric acid at constant temperature was studied iodometrically under pseudo-first order conditions with a very large excess of 5-oxoacids. Doubledistilled water (conductivity $<10 \mu \mathrm{S} \cdot \mathrm{cm}^{-1}$ ) was employed in all kinetic runs. All the chemicals used were $99.8 \%$ pure. The parent 5-oxoacid, namely, 5-oxo-5-phenylpentanoic acid (S1), and the phenyl-substituted 5-oxoacids (S2-S7) were prepared by Friedel-Crafts acylation of the substituted benzene with glutaric anhydride [15-19]. All the 5-oxoacids were crystallized twice from water and their purity was checked by their melting points and UV, IR, and NMR spectra. All absorption measurements were made with Shimadzu UV-visible spectrophotometer (MPS-5000) equipped with a temperature controller.

2.2. Kinetic Measurements. The reaction mixture, containing 5-oxoacid, catalyst, and sulphuric acid solutions, was thermally equilibrated and the reaction was initiated by the addition of temperature-equilibrated perborate solution of requisite concentration. The oxidation kinetics was followed iodometrically in aqueous acetic acid at constant temperature under pseudo-first order conditions by keeping the substrate in excess over the oxidant. The pseudo-first order rate constant was calculated from the slope of the linear plot of $\log [\text { perborate }]_{t}$ against time by the method of least squares. The error quoted in $k^{*}$ is the $95 \%$ confidence limit of a student's $t$-test. The progress of the oxidations was followed by iodometric determination of the oxidant. Freshly prepared solutions of oxoacids in purified acetic acid were used to avoid any possible side reactions.
TABLE 1: Pseudo-first order rate constant for perborate oxidation of substituted 5-oxo acids in presence of ruthenium(III) ${ }^{\mathrm{a}}$.

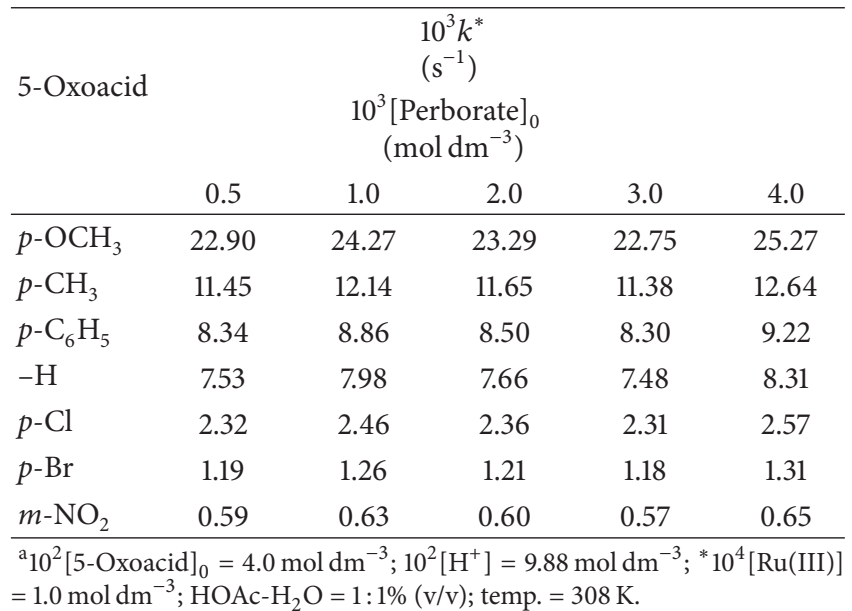

2.3. Reaction Stoichiometry and Product Analysis. Under the conditions [5-oxoacid $]_{0} \gg[\text { oxidant }]_{0} \gg[\mathrm{Ru}(\mathrm{III})]$, the stoichiometry of the catalytic reaction was determined by equilibrating reaction mixture of various [perborate]/[5oxoacid] ratios at $308 \mathrm{~K}$ for $12 \mathrm{~h}$, keeping all other reagents constant. Estimation of unconsumed perborate (iodometrically) revealed that one mole of 5-oxoacid consumed three moles of perborate (Scheme 2).

The products were extracted with ether, dried, and analyzed. Benzoic acid was identified by its melting point $\left(121^{\circ} \mathrm{C}\right)$. Then it was estimated quantitatively using UV-visible spectrophotometry with a standard curve at $\lambda_{\max }=235 \mathrm{~nm}$. Succinic acid was identified by its melting point $\left(185^{\circ} \mathrm{C}\right)$ and also tested with its characteristic spot test [20]. Identification of the products, namely, benzoic and succinic acids, was also made by comparing the $R_{f}$ values of the authentic samples.

\section{Results}

3.1. Effect of Concentrations. In acidic medium, ruthenium(III) chloride catalyzes perborate oxidation of 5oxoacids whereas cobalt(II) chloride, nickel(II) chloride, chromium(III) chloride, titanium(IV) chloride, cerium(III) chloride, thorium(IV) chloride, uranium(VI) chloride, copper(II) chloride, zinc(II) chloride, cadmium(II) chloride, mercury(II) chloride, aluminum(III) chloride, tin(II) chloride, lead(II) chloride, arsenic(III) chloride, antimony(III) chloride, bismuth(III) chloride, selenium(IV) chloride, and tellurium(IV) chloride do not.

Ruthenium(III) catalyzes perborate oxidation of substituted 5-oxoacids in acidic medium and the oxidation is first order with respect to perborate. Under the conditions [5oxoacid $]_{0} \gg[\text { oxidant }]_{0} \gg[\mathrm{Ru}(\mathrm{III})]$, plot of log [perborate $]_{t}$ versus time is linear at least up to $80 \%$ of the oxidation with correlation coefficient $(r)$ not less than 0.999 and standard error of estimate $(s d)$ not larger than 0.013 . The specific rate of oxidation in perborate remains constant when [perborate $]_{0}$ is increased by 8 -fold (Table 1 ). 
(1)

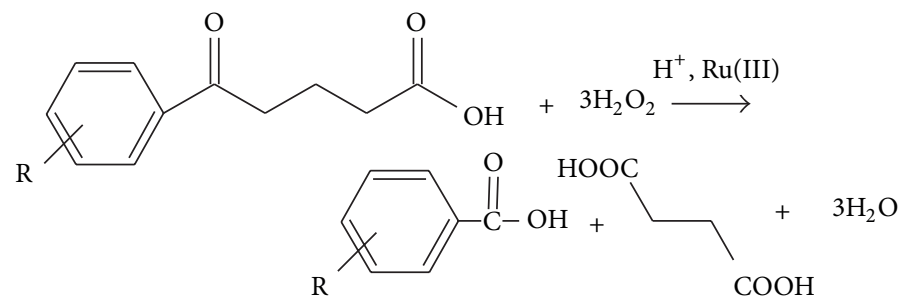

SCHEME 2

TABLE 2: Ruthenium(III) catalysis of perborate oxidation of substituted 5-oxo acids ${ }^{\mathrm{a}}$.

\begin{tabular}{|c|c|c|c|c|c|c|c|}
\hline \multirow[t]{3}{*}{ 5-Oxoacid } & \multicolumn{6}{|c|}{$\begin{array}{r}10^{5}[\text { Catalyst }] \\
\left(\mathrm{mol} \mathrm{dm}^{-3}\right)\end{array}$} & \\
\hline & \multicolumn{7}{|c|}{$\mathrm{Ru}(\mathrm{III})$} \\
\hline & 0 & 2.0 & 5.0 & 8.0 & 10.0 & 15.0 & 20.0 \\
\hline$p-\mathrm{OCH}_{3}$ & 8.63 & 12.43 & 16.20 & 20.34 & 23.28 & 29.83 & 35.88 \\
\hline$p-\mathrm{CH}_{3}$ & 4.31 & 6.21 & 8.10 & 10.16 & 11.64 & 14.91 & 17.93 \\
\hline$p-\mathrm{C}_{6} \mathrm{H}_{5}$ & 3.14 & 4.53 & 5.91 & 7.42 & 8.49 & 10.88 & 13.08 \\
\hline$-\mathrm{H}$ & 2.83 & 4.08 & 5.32 & 6.68 & 7.65 & 9.80 & 11.79 \\
\hline$p-\mathrm{Cl}$ & 0.87 & 1.27 & 1.64 & 2.06 & 2.35 & 3.02 & 3.63 \\
\hline$p-\mathrm{Br}$ & 0.44 & 0.63 & 0.83 & 1.04 & 1.20 & 1.53 & 1.85 \\
\hline$m-\mathrm{NO}_{2}$ & 0.21 & 0.31 & 0.41 & 0.51 & 0.59 & 0.76 & 0.91 \\
\hline
\end{tabular}

3.2. Effect of Catalyst. The oxidation is catalyzed by ruthenium(III) as well. The specific rate of oxidation in perborate increases with the increasing [Ru(III)] (Table 2). Plot of $k^{*}$, the specific oxidation rate in perborate in the presence of catalyst, versus $[\mathrm{Ru}(\mathrm{III})]$ is a straight line with a positive $y$ intercept (Figure $1 ; r=0.998$ and $0.9997, s d=1.34 \times 10^{-4}$ and $1.12 \times 10^{-4}$, slope $=44.1$ and $50.5 \mathrm{dm}^{3} \mathrm{~mol}^{-1} \mathrm{~s}^{-1}$, and intercept $=3.10 \times 10^{-3}$ and $2.87 \times 10^{-3} \mathrm{~s}^{-1}$ for ruthenium(III).

The oxidation proceeds in the absence of catalysts also and the uncatalyzed oxidation is first order in the oxidant. Plot of $\log$ [perborate $]_{t}$ versus time is linear. Also, the pseudofirst order rate constant of the uncatalyzed oxidation $\left(k^{\prime}\right)$ remains constant when the $[\text { perborate }]_{0}$ is varied 8 -fold. The $y$-intercepts of $k^{*}$ versus [Ru(III)] plots are equal to the specific rate of uncatalyzed oxidation. The difference $\left(k^{*}-\right.$ $\left.k^{\prime}\right)$ is the specific rate of ruthenium(III) catalyzed oxidation, and the ruthenium(III) catalyzed oxidation is first order with respect to the catalyst.

At fixed [perborate $]_{0},\left[\mathrm{H}^{+}\right]$and $[\mathrm{Ru}(\mathrm{III})]$, the specific rate of ruthenium(III) catalyzed oxidation increases but less rapidly with the increasing [5-oxoacid $]_{0}$ (Table 3 ). The dependence of specific rate of ruthenium(III) catalyzed oxidation on [5-oxoacid $]_{0}$ at 298, 308, and $318 \mathrm{~K}$ is the MichaelisMenten type (Figure 2). The double reciprocal plot of rate versus [5-oxoacid $]_{0}$ and the statistically balanced Hanes plot $\left([5 \text {-oxoacid }]_{0} /\left(k^{*}-k^{\prime}\right)\right.$ versus $\left.[5 \text {-oxoacid }]_{0}\right)$ are linear (Figure 3; Hanes plot: slope $=1.67 \times 10^{2}, 1.01 \times 10^{2}$, and $79.2 \mathrm{~s}$,

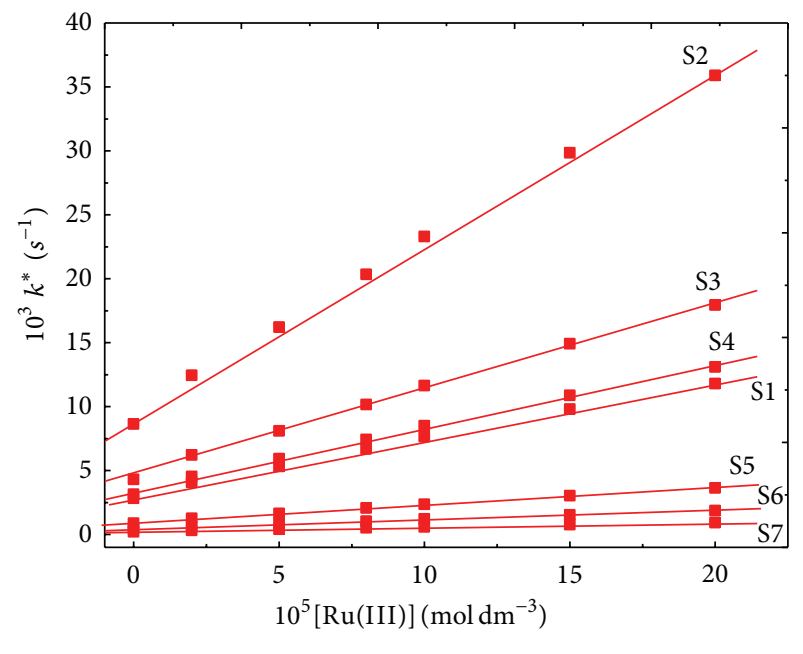

- $\mathrm{Ru}(\mathrm{III})$

FIGURE 1: Linear dependence of $k^{*}$ on [catalyst] under the conditions of Table 2. (S1) - H, (S2) $4^{\prime}$-methoxy, (S3) $4^{\prime}$-methyl, (S4) $4^{\prime}$ phenyl, (S5) $4^{\prime}$-Chloro, (S6) $4^{\prime}$-bromo, (S7) $3^{\prime}$-nitro.

intercept $=9.77,3.82$, and $1.62 \mathrm{~mol} \mathrm{dm}^{-3} \mathrm{~s}, r=0.971,0.993$, and $0.982, s d=1.52,0.388$, and 0.446 at 298,308 , and $318 \mathrm{~K}$, resp.).

The rate of ruthenium(III) catalyzed oxidation is independent of $\left[\mathrm{H}^{+}\right]$of the medium. At fixed [perborate $]_{0},[5-$ oxoacid $]_{0}$, and $[\mathrm{Ru}(\mathrm{III})],\left(k^{*}-k^{\prime}\right)$ remains constant when $\left[\mathrm{H}^{+}\right]$is varied twentyfold. Table 4 presents constancy of specific reaction rate at different acidities. But, both the catalyzed and uncatalyzed oxidations occur only in acidic medium.

3.3. Influence of Salt Effect and Solvent Polarity. The variation of oxidation rate with ionic strength of the medium (adjusted with $\mathrm{KNO}_{3}$ ) is quite small at low $\left[\mathrm{H}^{+}\right]$but appreciable at high $\left[\mathrm{H}^{+}\right]$(Table 5).

At low and high acidities, the ruthenium(III) catalyzed oxidation rate increases with the decreasing dielectric constant of the reaction medium; the dielectric constant was decreased by the addition of acetic acid (Table 6).

3.4. Effect of Hydrogen Peroxide. Under identical conditions, the rates of ruthenium(III) catalyzed oxidation of 5-oxoacids by perborate and hydrogen peroxide are almost the same (Table 7). 
TABle 3: Rate dependence on [5-oxoacid $]_{0}{ }^{\mathrm{a}}$.

(a)

\begin{tabular}{|c|c|c|c|c|c|c|c|c|c|c|c|c|}
\hline \multirow[t]{2}{*}{ 5-Oxoacid } & \multicolumn{5}{|c|}{$\begin{array}{c}10^{3} k^{*} \\
\left(\mathrm{~s}^{-1}\right) \\
10^{2}[5-\text { Oxoacid }]_{0} \\
\left(\mathrm{~mol} \mathrm{dm}^{-3}\right) \\
298 \mathrm{~K}\end{array}$} & \multirow[b]{2}{*}{10.0} & \multirow[b]{2}{*}{1.0} & \multicolumn{4}{|c|}{$\begin{array}{c}10^{3}\left(k^{*}-k^{\prime}\right) \\
\left(\mathrm{s}^{-1}\right) \\
298 \mathrm{~K}\end{array}$} & \multirow[b]{2}{*}{10.0} \\
\hline & 1.0 & 3.0 & 4.0 & 6.0 & 8.0 & & & 3.0 & 4.0 & 6.0 & 8.0 & \\
\hline$p-\mathrm{OCH}_{3}$ & $\begin{array}{c}4.92 \\
(2.13)\end{array}$ & $\begin{array}{l}10.03 \\
(4.75)\end{array}$ & $\begin{array}{c}12.31 \\
(4.19)\end{array}$ & $\begin{array}{c}16.23 \\
(6.57)\end{array}$ & $\begin{array}{c}18.91 \\
(8.67)\end{array}$ & $\begin{array}{c}21.46 \\
(10.07)\end{array}$ & 2.78 & 5.28 & 8.11 & 9.66 & 10.24 & 11.39 \\
\hline$p-\mathrm{CH}_{3}$ & $\begin{array}{c}2.45 \\
(1.07)\end{array}$ & $\begin{array}{c}5.01 \\
(2.37)\end{array}$ & $\begin{array}{c}6.15 \\
(2.09)\end{array}$ & $\begin{array}{c}8.11 \\
(3.29)\end{array}$ & $\begin{array}{c}9.45 \\
(4.33)\end{array}$ & $\begin{array}{l}10.73 \\
(5.03)\end{array}$ & 1.38 & 2.64 & 4.05 & 4.83 & 5.12 & 5.69 \\
\hline$p-\mathrm{C}_{6} \mathrm{H}_{5}$ & $\begin{array}{c}1.78 \\
(0.78)\end{array}$ & $\begin{array}{c}3.65 \\
(1.73)\end{array}$ & $\begin{array}{c}4.48 \\
(1.53)\end{array}$ & $\begin{array}{c}5.91 \\
(2.39)\end{array}$ & $\begin{array}{c}6.89 \\
(3.16)\end{array}$ & $\begin{array}{c}7.83 \\
(3.67)\end{array}$ & 1.01 & 1.92 & 2.95 & 3.51 & 3.73 & 4.15 \\
\hline$-\mathrm{H}$ & $\begin{array}{c}1.61 \\
(0.70)\end{array}$ & $\begin{array}{c}3.29 \\
(1.56)\end{array}$ & $\begin{array}{c}4.04 \\
(1.38)\end{array}$ & $\begin{array}{c}5.33 \\
(2.16)\end{array}$ & $\begin{array}{c}6.21 \\
(2.85)\end{array}$ & $\begin{array}{c}7.05 \\
(3.31)\end{array}$ & 0.91 & 1.73 & 2.66 & 3.17 & 3.36 & 3.74 \\
\hline$p-\mathrm{Cl}$ & $\begin{array}{c}0.49 \\
(0.22)\end{array}$ & $\begin{array}{c}1.01 \\
(0.48)\end{array}$ & $\begin{array}{c}1.24 \\
(0.43)\end{array}$ & $\begin{array}{c}1.64 \\
(0.67)\end{array}$ & $\begin{array}{c}1.91 \\
(0.88)\end{array}$ & $\begin{array}{c}2.17 \\
(1.02)\end{array}$ & 0.27 & 0.53 & 0.81 & 0.97 & 1.03 & 1.15 \\
\hline$p-\mathrm{Br}$ & $\begin{array}{c}0.25 \\
(0.11)\end{array}$ & $\begin{array}{c}0.51 \\
(0.25)\end{array}$ & $\begin{array}{c}0.63 \\
(0.22)\end{array}$ & $\begin{array}{c}0.83 \\
(0.34)\end{array}$ & $\begin{array}{c}0.97 \\
(0.45)\end{array}$ & $\begin{array}{c}1.10 \\
(0.52)\end{array}$ & 0.14 & 0.26 & 0.41 & 0.49 & 0.52 & 0.58 \\
\hline$m-\mathrm{NO}_{2}$ & $\begin{array}{c}0.12 \\
(0.06)\end{array}$ & $\begin{array}{c}0.25 \\
(0.12)\end{array}$ & $\begin{array}{c}0.31 \\
(0.11)\end{array}$ & $\begin{array}{c}0.41 \\
(0.17)\end{array}$ & $\begin{array}{c}0.48 \\
(0.22)\end{array}$ & $\begin{array}{c}0.54 \\
(0.26)\end{array}$ & 0.06 & 0.13 & 0.20 & 0.24 & 0.25 & 0.28 \\
\hline
\end{tabular}

(b)

\begin{tabular}{|c|c|c|c|c|c|c|c|c|c|c|c|c|}
\hline \multirow[t]{2}{*}{ 5-Oxoacid } & \multicolumn{5}{|c|}{$\begin{array}{c}10^{3} k^{*} \\
\left(\mathrm{~s}^{-1}\right) \\
10^{2}[5-\text { Oxoacid }]_{0} \\
\left(\mathrm{~mol} \mathrm{dm}^{-3}\right) \\
308 \mathrm{~K}\end{array}$} & \multirow[b]{2}{*}{10.0} & \multirow[b]{2}{*}{2.0} & \multicolumn{4}{|c|}{$\begin{array}{c}10^{3}\left(k^{*}-k^{\prime}\right) \\
\left(\mathrm{s}^{-1}\right) \\
308 \mathrm{~K}\end{array}$} & \multirow[b]{2}{*}{10.0} \\
\hline & 2.0 & 3.0 & 4.0 & 6.0 & 8.0 & & & 3.0 & 4.0 & 6.0 & 8.0 & \\
\hline$p-\mathrm{OCH}_{3}$ & $\begin{array}{l}14.98 \\
(4.78)\end{array}$ & $\begin{array}{l}20.46 \\
(7.66)\end{array}$ & $\begin{array}{l}24.26 \\
(8.64)\end{array}$ & $\begin{array}{c}31.62 \\
(11.95)\end{array}$ & $\begin{array}{c}36.18 \\
(16.21)\end{array}$ & $\begin{array}{c}40.14 \\
(18.86)\end{array}$ & 10.12 & 12.79 & 15.62 & 19.67 & 20.09 & 21.37 \\
\hline$p-\mathrm{CH}_{3}$ & $\begin{array}{c}7.48 \\
(2.39)\end{array}$ & $\begin{array}{l}10.22 \\
(3.83)\end{array}$ & $\begin{array}{c}12.13 \\
(4.32)\end{array}$ & $\begin{array}{c}15.81 \\
(5.98)\end{array}$ & $\begin{array}{l}18.08 \\
(8.11)\end{array}$ & $\begin{array}{l}20.06 \\
(9.43)\end{array}$ & 5.05 & 6.39 & 7.81 & 9.83 & 10.04 & 10.68 \\
\hline$p-\mathrm{C}_{6} \mathrm{H}_{5}$ & $\begin{array}{c}5.46 \\
(1.74)\end{array}$ & $\begin{array}{c}7.46 \\
(2.79)\end{array}$ & $\begin{array}{c}8.85 \\
(3.15)\end{array}$ & $\begin{array}{c}11.54 \\
(4.36)\end{array}$ & $\begin{array}{l}13.20 \\
(5.92)\end{array}$ & $\begin{array}{l}14.64 \\
(6.88)\end{array}$ & 3.68 & 4.66 & 5.70 & 7.17 & 7.33 & 7.79 \\
\hline$-\mathrm{H}$ & $\begin{array}{c}4.92 \\
(1.57)\end{array}$ & $\begin{array}{c}6.72 \\
(2.52)\end{array}$ & $\begin{array}{c}7.97 \\
(2.84)\end{array}$ & $\begin{array}{l}10.39 \\
(3.93)\end{array}$ & $\begin{array}{l}11.89 \\
(5.33)\end{array}$ & $\begin{array}{c}13.19 \\
(6.20)\end{array}$ & 3.32 & 4.20 & 5.13 & 6.46 & 6.60 & 7.02 \\
\hline$p-\mathrm{Cl}$ & $\begin{array}{c}1.51 \\
(0.49)\end{array}$ & $\begin{array}{c}2.07 \\
(0.78)\end{array}$ & $\begin{array}{c}2.45 \\
(0.88)\end{array}$ & $\begin{array}{c}3.20 \\
(1.21)\end{array}$ & $\begin{array}{c}3.66 \\
(1.65)\end{array}$ & $\begin{array}{c}4.06 \\
(1.91)\end{array}$ & 1.02 & 1.28 & 1.58 & 1.98 & 2.03 & 2.16 \\
\hline$p-\mathrm{Br}$ & $\begin{array}{c}0.77 \\
(0.25)\end{array}$ & $\begin{array}{c}1.05 \\
(0.39)\end{array}$ & $\begin{array}{c}1.25 \\
(0.45)\end{array}$ & $\begin{array}{l}1.63 \\
(0.62)\end{array}$ & $\begin{array}{c}1.86 \\
(0.84)\end{array}$ & $\begin{array}{c}2.07 \\
(0.98)\end{array}$ & 0.51 & 0.65 & 0.80 & 1.01 & 1.03 & 1.10 \\
\hline$m-\mathrm{NO}_{2}$ & $\begin{array}{c}0.38 \\
(0.12) \\
\end{array}$ & $\begin{array}{c}0.52 \\
(0.19) \\
\end{array}$ & $\begin{array}{c}0.62 \\
(0.22) \\
\end{array}$ & $\begin{array}{c}0.81 \\
(0.31) \\
\end{array}$ & $\begin{array}{c}0.92 \\
(0.42) \\
\end{array}$ & $\begin{array}{c}1.02 \\
(0.49) \\
\end{array}$ & 0.25 & 0.32 & 0.39 & 0.50 & 0.51 & 0.54 \\
\hline \multicolumn{13}{|c|}{ (c) } \\
\hline \multirow[t]{2}{*}{ 5-Oxoacid } & & & $\begin{array}{r}1 \\
10^{2}[5-(\mathrm{m} \\
\end{array}$ & $\begin{array}{l}\text { l) } \\
\text { oacid }]_{0} \\
\left.\mathrm{dm}^{-3}\right) \\
\mathrm{K}\end{array}$ & & & \multicolumn{6}{|c|}{$\begin{array}{c}10^{3}\left(k^{*}-k^{\prime}\right) \\
\left(\mathrm{s}^{-1}\right) \\
318 \mathrm{~K}\end{array}$} \\
\hline & 1.0 & 2.0 & 3.0 & 4.0 & 6.0 & 8.0 & 1.0 & 2.0 & 3.0 & 4.0 & 6.0 & 8.0 \\
\hline$p-\mathrm{OCH}_{3}$ & $\begin{array}{l}16.05 \\
(5.75)\end{array}$ & $\begin{array}{l}29.34 \\
(9.25)\end{array}$ & $\begin{array}{c}36.48 \\
(12.89)\end{array}$ & $\begin{array}{l}44.70 \\
(15.51)\end{array}$ & $\begin{array}{c}51.08 \\
(22.99)\end{array}$ & $\begin{array}{c}56.56 \\
(26.89)\end{array}$ & 10.30 & 20.09 & 23.47 & 29.22 & 27.97 & 29.74 \\
\hline$p-\mathrm{CH}_{3}$ & $\begin{array}{c}8.02 \\
(2.87)\end{array}$ & $\begin{array}{l}14.66 \\
(4.62)\end{array}$ & $\begin{array}{l}18.24 \\
(6.45)\end{array}$ & $\begin{array}{l}22.34 \\
(7.76)\end{array}$ & $\begin{array}{c}25.54 \\
(11.49)\end{array}$ & $\begin{array}{c}28.28 \\
(13.44)\end{array}$ & 5.15 & 10.04 & 11.73 & 14.60 & 13.98 & 14.86 \\
\hline
\end{tabular}


(c) Continued.

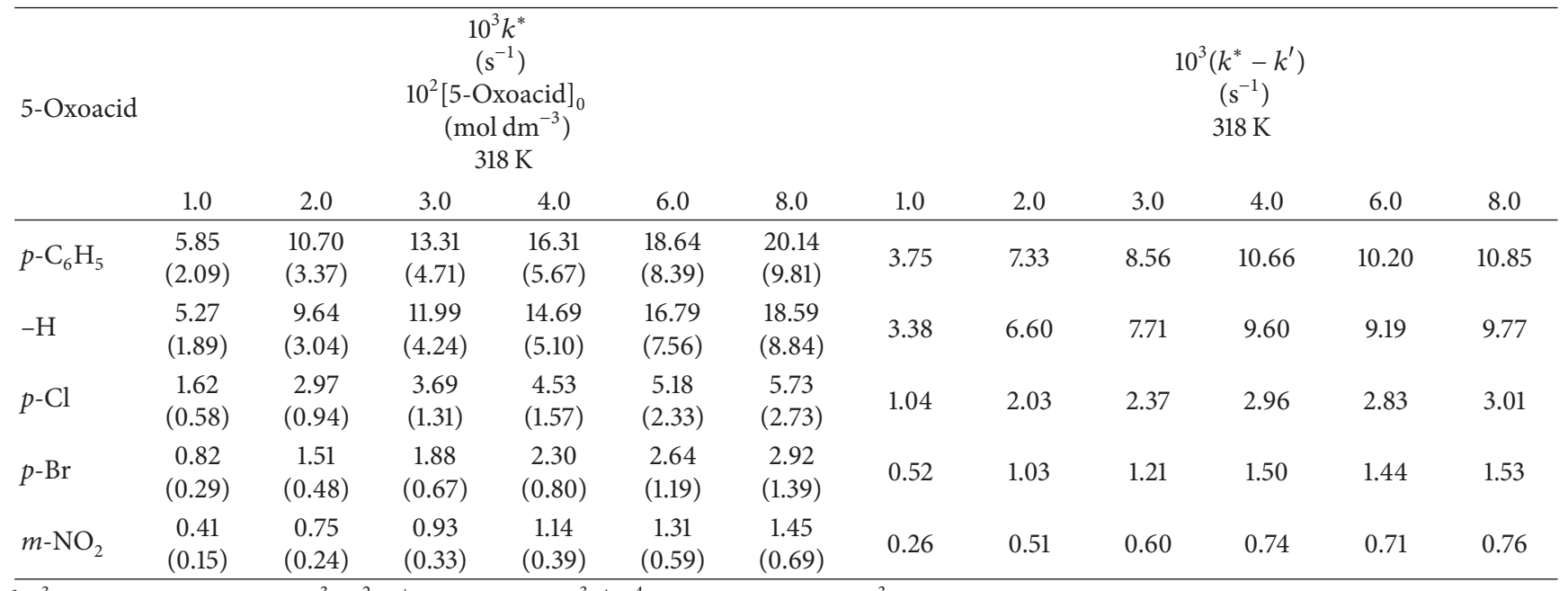

${ }^{\mathrm{a}} 10^{3}[\text { Perborate }]_{0}=1.0 \mathrm{~mol} \mathrm{dm}^{-3} ; 10^{2}\left[\mathrm{H}^{+}\right]=9.88 \mathrm{~mol} \mathrm{dm}^{-3} ;{ }^{*} 10^{4}[\mathrm{Ru}(\mathrm{III})]=1.0 \mathrm{~mol} \mathrm{dm}^{-3} ; \mathrm{HOAc}-\mathrm{H}_{2} \mathrm{O}=1: 1 \%(\mathrm{v} / \mathrm{v})$.

Parentheses values indicate the rate constant $\left(k^{\prime}, \mathrm{s}^{-1}\right)$ of uncatalyzed reactions.

TABLE 4: Oxidation rates at different acidities ${ }^{\mathrm{a}}$.

\begin{tabular}{|c|c|c|c|c|c|c|c|c|c|c|c|c|c|c|}
\hline \multirow[t]{2}{*}{ 5-Oxoacid } & \multicolumn{6}{|c|}{$\begin{array}{c}10^{3} k^{*} \\
\left(\mathrm{~s}^{-1}\right) \\
10^{2}\left[\mathrm{H}^{+}\right] \\
\left(\mathrm{mol} \mathrm{dm} \mathrm{dm}^{-3}\right)\end{array}$} & \multirow[b]{2}{*}{19.9} & \multirow[b]{2}{*}{0.88} & \multirow[b]{2}{*}{1.88} & \multicolumn{3}{|c|}{$\begin{array}{c}10^{3}\left(k^{*}-k^{\prime}\right) \\
\left(\mathrm{s}^{-1}\right)\end{array}$} & \multirow[b]{2}{*}{14.9} & \multirow[b]{2}{*}{19.9} \\
\hline & 0.88 & 1.88 & 3.88 & 6.88 & 9.88 & 14.9 & & & & 3.88 & 6.88 & 9.88 & & \\
\hline$p-\mathrm{OCH}_{3}$ & $\begin{array}{l}20.27 \\
(4.11)\end{array}$ & $\begin{array}{l}21.45 \\
(4.41)\end{array}$ & $\begin{array}{l}22.86 \\
(5.93)\end{array}$ & $\begin{array}{l}23.65 \\
(7.42)\end{array}$ & $\begin{array}{l}24.26 \\
(8.64)\end{array}$ & $\begin{array}{c}27.33 \\
(11.56)\end{array}$ & $\begin{array}{c}28.70 \\
(12.80)\end{array}$ & 16.16 & 17.04 & 16.93 & 16.23 & 15.62 & 15.78 & 15.90 \\
\hline$p-\mathrm{CH}_{3}$ & $\begin{array}{c}9.13 \\
(2.05)\end{array}$ & $\begin{array}{c}11.12 \\
(2.21)\end{array}$ & $\begin{array}{l}11.43 \\
(2.97)\end{array}$ & $\begin{array}{l}11.82 \\
(3.71)\end{array}$ & $\begin{array}{c}12.13 \\
(4.32)\end{array}$ & $\begin{array}{l}13.66 \\
(5.78)\end{array}$ & $\begin{array}{c}14.35 \\
(6.40)\end{array}$ & 7.08 & 8.92 & 8.46 & 8.46 & 7.81 & 7.88 & 7.94 \\
\hline$p-\mathrm{C}_{6} \mathrm{H}_{5}$ & $\begin{array}{c}6.66 \\
(1.44)\end{array}$ & $\begin{array}{c}8.12 \\
(1.61)\end{array}$ & $\begin{array}{c}8.34 \\
(2.17)\end{array}$ & $\begin{array}{c}8.63 \\
(2.71)\end{array}$ & $\begin{array}{c}8.85 \\
(3.15)\end{array}$ & $\begin{array}{c}9.97 \\
(4.22)\end{array}$ & $\begin{array}{l}10.47 \\
(4.67)\end{array}$ & 5.16 & 6.51 & 6.17 & 6.17 & 5.70 & 5.75 & 5.80 \\
\hline$-\mathrm{H}$ & $\begin{array}{c}6.00 \\
(1.35)\end{array}$ & $\begin{array}{c}7.31 \\
(1.45)\end{array}$ & $\begin{array}{c}7.51 \\
(1.95)\end{array}$ & $\begin{array}{c}7.77 \\
(2.44)\end{array}$ & $\begin{array}{c}7.97 \\
(2.84)\end{array}$ & $\begin{array}{c}8.98 \\
(3.80)\end{array}$ & $\begin{array}{c}9.43 \\
(4.21)\end{array}$ & 4.65 & 5.86 & 5.56 & 5.33 & 5.13 & 5.18 & 5.22 \\
\hline$p-\mathrm{Cl}$ & $\begin{array}{c}1.84 \\
(0.42)\end{array}$ & $\begin{array}{c}2.25 \\
(0.45)\end{array}$ & $\begin{array}{c}2.31 \\
(0.60)\end{array}$ & $\begin{array}{c}2.39 \\
(0.75)\end{array}$ & $\begin{array}{c}2.45 \\
(0.88)\end{array}$ & $\begin{array}{c}2.77 \\
(1.17)\end{array}$ & $\begin{array}{c}2.90 \\
(1.29)\end{array}$ & 1.43 & 1.80 & 1.71 & 1.64 & 1.58 & 1.59 & 1.60 \\
\hline$p-\mathrm{Br}$ & $\begin{array}{c}0.94 \\
(0.21)\end{array}$ & $\begin{array}{c}1.14 \\
(0.23)\end{array}$ & $\begin{array}{c}1.83 \\
(0.31)\end{array}$ & $\begin{array}{c}1.22 \\
(0.38)\end{array}$ & $\begin{array}{c}1.25 \\
(0.45)\end{array}$ & $\begin{array}{c}1.41 \\
(0.59)\end{array}$ & $\begin{array}{c}1.48 \\
(0.66)\end{array}$ & 0.72 & 0.91 & 0.87 & 0.83 & 0.80 & 0.81 & 0.81 \\
\hline$m-\mathrm{NO}_{2}$ & $\begin{array}{c}0.46 \\
(0.11)\end{array}$ & $\begin{array}{c}0.56 \\
(0.11)\end{array}$ & $\begin{array}{c}0.58 \\
(0.15)\end{array}$ & $\begin{array}{c}0.60 \\
(0.18)\end{array}$ & $\begin{array}{c}0.62 \\
(0.22)\end{array}$ & $\begin{array}{c}0.69 \\
(0.33)\end{array}$ & $\begin{array}{c}0.73 \\
(0.33)\end{array}$ & 0.35 & 0.45 & 0.43 & 0.42 & 0.40 & 0.36 & 0.40 \\
\hline
\end{tabular}

${ }^{\mathrm{a}} 10^{3}[\text { Perborate }]_{0}=1.0 \mathrm{~mol} \mathrm{dm}^{-3} ; 10^{2}[5 \text {-oxoacid }]_{0}=4.0 \mathrm{~mol} \mathrm{dm}^{-3} ;{ }^{*} 10^{4}[\mathrm{Ru}(\mathrm{III})]=1.0 \mathrm{~mol} \mathrm{dm}^{-3} ; \mathrm{HOAc}-\mathrm{H}_{2} \mathrm{O}=1: 1 \%(\mathrm{v} / \mathrm{v}) ;$ Temp. $=308 \mathrm{~K}$.

Parentheses values indicate the rate constant $\left(k^{\prime}, \mathrm{s}^{-1}\right)$ of uncatalyzed reactions.

3.5. Influence of Metaborate and Orthoboric Acid. Boric acid or borate neither retards nor enhances the oxidation. Initial addition of metaborate or boric acid to the reaction solution has insignificant effect on the rates of oxidation by perborate and hydrogen peroxide (Table 8 ). The reaction solution, when the oxidation is in progress, does not induce polymerization of vinyl monomer acrylonitrile. Also, initial addition of acrylonitrile to the reaction mixture fails to suppress the oxidation rate (Table 8 ). During the course of oxidation, the reaction solution does not show an ESR signal (Bruker Xband) for any radical.

3.6. Effect of Temperature. The oxidation reactions were studied in the temperature range of 298-318 K. Activation energy (Ea) of the reactions was calculated from the least-square slopes of linear Arrhenius plots (Figure 4; $r \geq 0.96 ; s \leq 0.02$ ) of $\log k$ versus $1 / T$. The related thermodynamic parameters, namely, enthalpy of activation $\left(\Delta H^{\#}\right)$, entropy of activation $\left(\Delta S^{\#}\right)$, and Gibbs free-energy of activation $\left(\Delta G^{\#}\right)$ calculated using appropriate equations, are presented in Table 9.

3.7. Isokinetic Relationships. The plot between $\Delta H^{\#}$ and $\Delta S^{\#}$ is linear (Figure 5; $r \geq 0.97 ; s \leq 0.029$ ) and the isokinetic temperature $(\beta)$ obtained is $355.9 \mathrm{~K}$. The $\beta$ calculated from Exner's plot (Figure 6; $r \geq 0.987 ; s \leq 0.04$ ) of $\log k_{318 \mathrm{~K}}$ against $\log k_{308 \mathrm{~K}}$ is $353.7 \mathrm{~K}$, which is in good agreement with the value obtained from the $\Delta H^{\#}$ against $\Delta S^{\#}$ plot. The isokinetic relationship in the present study implies that all the 5-oxoacids undergo oxidation by the same mechanism [21]. 

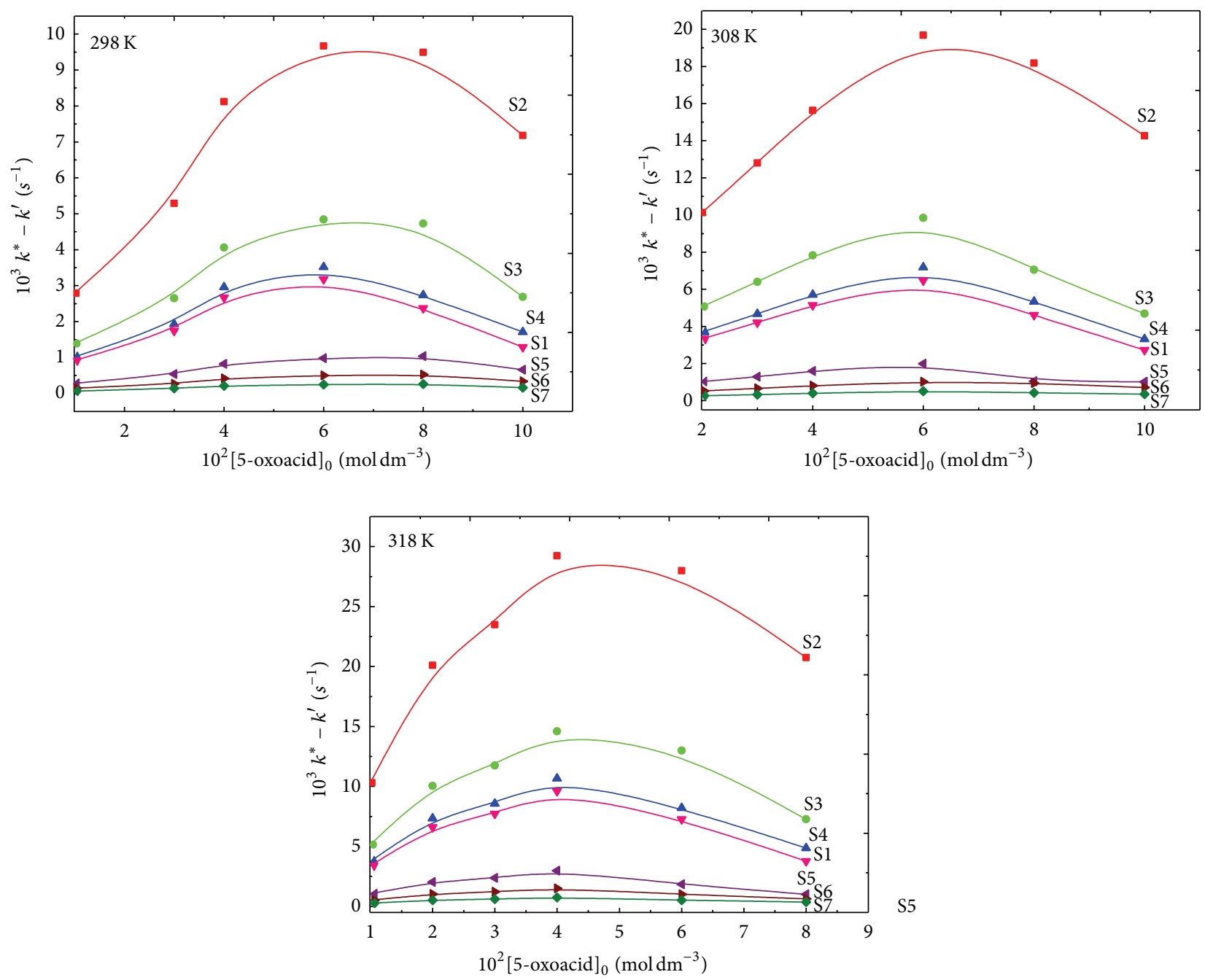

Figure 2: Variation of $k^{*}-k^{\prime}$ with [5-oxoacid] $]_{0}$ under the conditions of Table 3 at (a) $298 \mathrm{~K}$, (b) $308 \mathrm{~K}$, and (c) $318 \mathrm{~K}$. (S1) -H, (S2) $4^{\prime}$-methoxy, (S3) $4^{\prime}$-methyl, (S4) $4^{\prime}$-phenyl, (S5) $4^{\prime}$-chloro, (S6) $4^{\prime}$-bromo, (S7) $3^{\prime}$-nitro.

(2)<smiles>[R]c1ccc(C(=O)CCCC(=O)OC([R])(Cl)Cl)cc1</smiles><smiles>[R]c1ccc(/C(O)=C/CCC(=O)O)cc1</smiles>

Scheme 3

\section{Discussion}

4.1. Active Species of Reactants. The oxoacid is a weak acid $\left(p K_{a}=5.77\right.$ at $40^{\circ} \mathrm{C}$ in aqueous solution) [22], and the undissociated form of the substrate can be taken as the only form in acidic media. In acid solutions, 5-oxoacid undergoes keto-enol tautomerism (Scheme 3).

In crystalline state sodium perborate exists as a dimer with anionic formula: $\mathrm{B}_{2}\left(\mathrm{O}_{2}\right)_{2}(\mathrm{OH})_{4}{ }^{2-}$. But in aqueous solution it affords hydrogen peroxide $[1,23]$. Although perboric acid is reported to exist in equilibrium with hydrogen peroxide, the equilibrium constant reveals that, even in the presence of large excess of boric acid, the concentration of perboric acid is insignificant [24]. For example, at $\left[\mathrm{H}_{3} \mathrm{BO}_{3}\right]_{0}=$ $0.01 \mathrm{~mol} \mathrm{dm}^{-3},\left[(\mathrm{HO})_{2} \mathrm{BOOH}\right] /\left[\mathrm{H}_{2} \mathrm{O}_{2}\right]=1 \times 10^{-4}$. The similar rates of ruthenium(III) catalyzed oxidation of 5-oxoacids by perborate and hydrogen peroxide, under identical conditions, indicate that hydrogen peroxide is the oxidizing species of perborate.

Mechanism. Ruthenium(III) oxidizes 5-oxoacids to benzoic acid. Hence, one of the possible mechanisms is oxidation of 

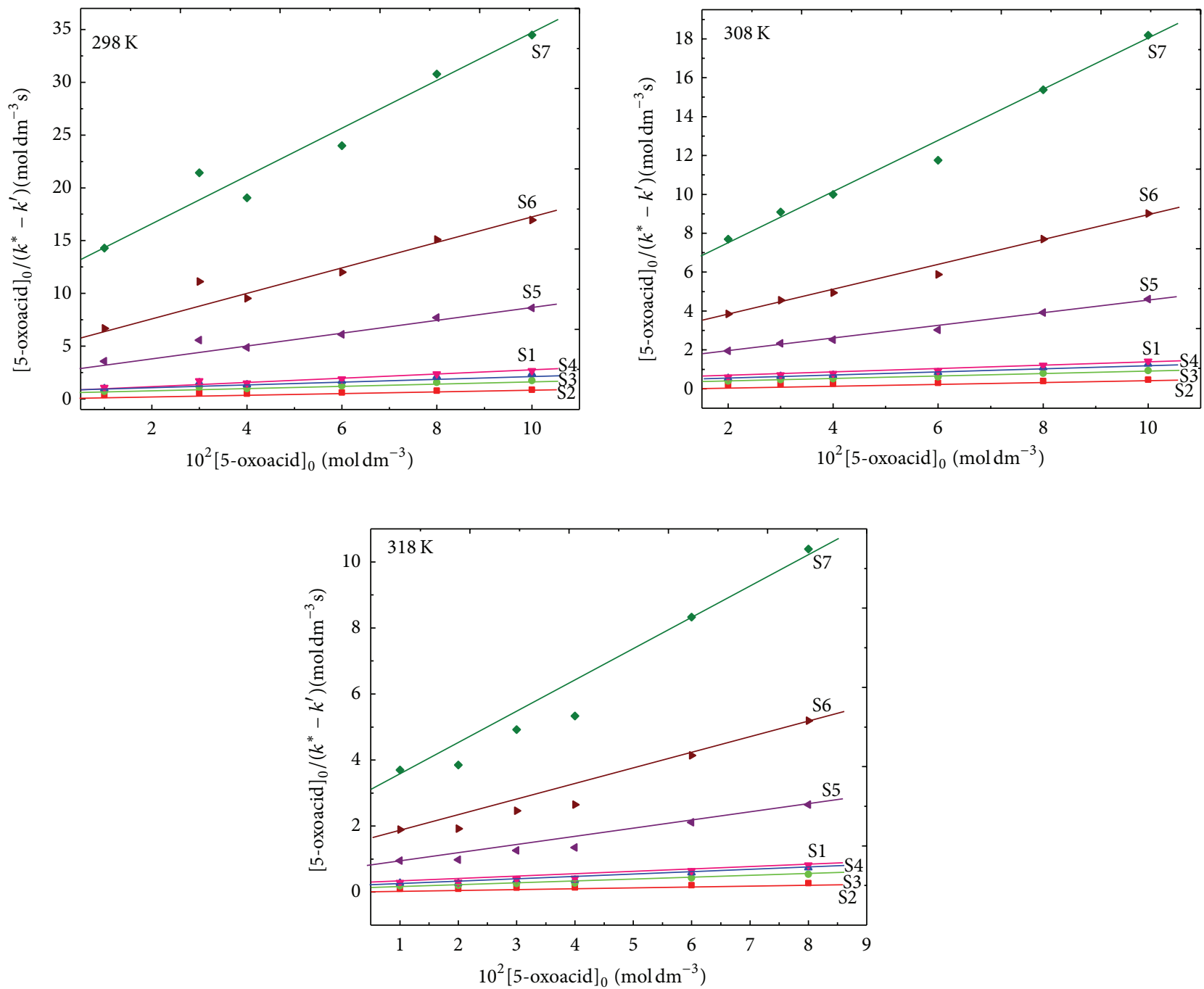

Figure 3: Hanes plot under the conditions of Table 3 at (a) $298 \mathrm{~K}$, (b) $308 \mathrm{~K}$, and (c) $318 \mathrm{~K}$. (S1) -H, (S2) $4^{\prime}$-methoxy, (S3) $4^{\prime}$-methyl, (S4) $4^{\prime}$-phenyl, (S5) $4^{\prime}$-chloro, (S6) $4^{\prime}$-bromo, (S7) $3^{\prime}$-nitro.

ruthenium(III) followed by the reduction of ruthenium(III) by 5 -oxoacids. Chemical tests confirm the presence of ruthenium(III) and the absence of ruthenium(III) in the reaction solution when the oxidation is in progress. Formation of the Michaelis-Menten type complex between ruthenium(III) and 5-oxoacid is also unlikely.

Another possible mechanism is of the Fenton type. But this mechanism is also ruled out as the oxidation is not through radical pathway. ESR spectral study of the reaction solution, while the oxidation is in progress, does not show the presence of any radical. The ruthenium(III) catalyzed oxidation is insensitive to the addition of vinyl monomer and the reaction solution fails to initiate polymerization of acrylonitrile. Although $\mathrm{Ru}_{2} \mathrm{O}_{2}$ are suggested as intermediates in ruthenium(III) catalyzed decomposition of hydrogen peroxide they are not considered as the active oxidizing species in the present work [25]. If they were to be the oxidizing species, contrary to the experimental findings, the oxidation should be second order with respect to the catalyst. In acidic solution hydrogen peroxide affords ruthenium(III) peroxo species $[26,27]$. This species contains ruthenium and peroxide in the ratio $1: 1$ and is formulated as $\mathrm{Ru}\left(\mathrm{O}_{2} \mathrm{H}\right)^{2+}$. The following mechanism accounts for the observed kinetic results (Scheme 4).

Rate Law. The rate law for the catalyzed oxidation is

$$
\begin{aligned}
& \frac{-d \text { [perborate }]}{d t} \\
& =\frac{K_{1} K_{2} k_{3}[\mathrm{Ru}(\mathrm{III})][5 \text {-oxoacid }][\text { perborate }]}{\left(1+K_{2}[5 \text {-oxoacid }]\right)} .
\end{aligned}
$$

The specific rate in perborate of the catalyzed oxidation is

$$
k^{*}-k^{\prime}=\frac{K_{1} K_{2} k_{3}[\mathrm{Ru}(\mathrm{III})][5 \text {-oxoacid }]}{\left(1+K_{2}[5 \text {-oxoacid }]\right)}
$$




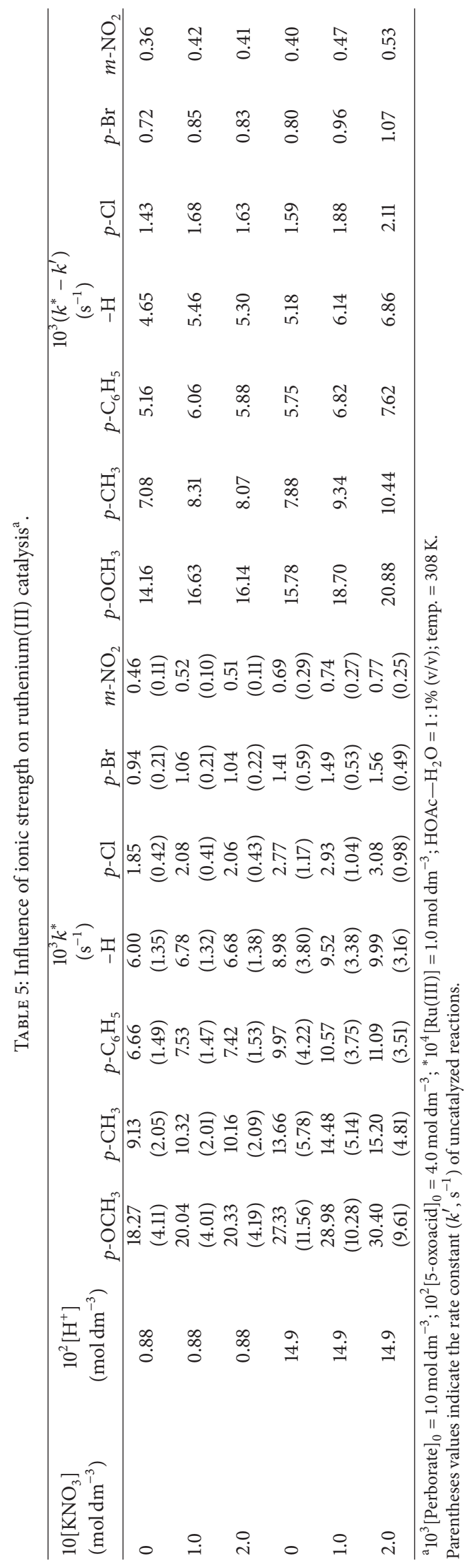




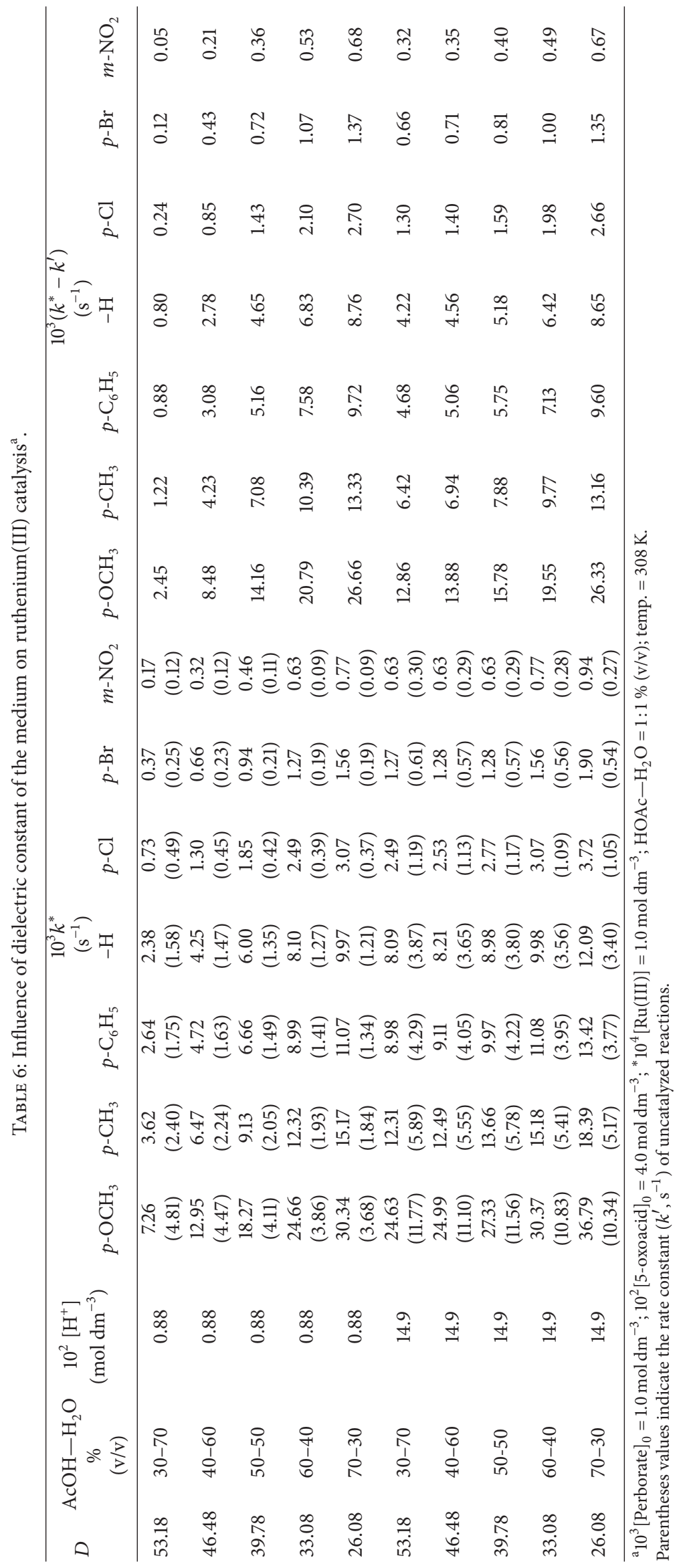




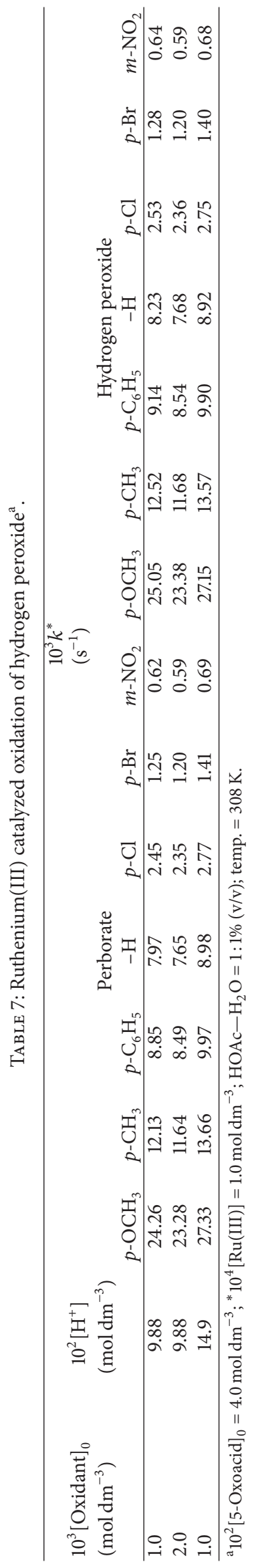




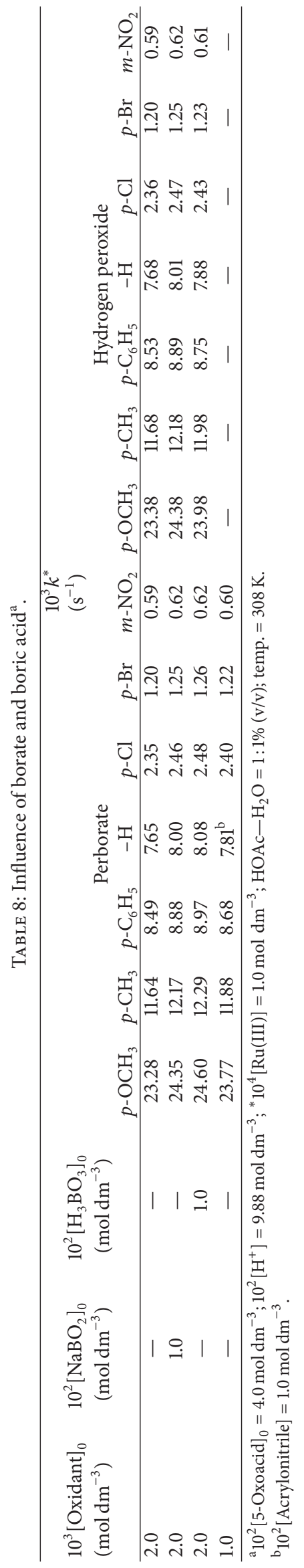


TABLE 9: Values of rate constant at different temperatures and activation parameters ${ }^{\mathrm{a}}$.

\begin{tabular}{|c|c|c|c|c|c|c|}
\hline \multirow[t]{2}{*}{ 5-Oxoacid } & \multicolumn{3}{|c|}{$\begin{array}{c}10^{3}\left(k^{*}-k^{\prime}\right) \\
\left(\mathrm{s}^{-1}\right)\end{array}$} & \multirow{2}{*}{$\begin{array}{c}\Delta H^{\#} \\
\left(\mathrm{~kJ} \mathrm{~mol}{ }^{-1}\right)\end{array}$} & \multirow{2}{*}{$\begin{array}{c}\Delta S^{\#} \\
\left(\mathrm{~J} \mathrm{~K}^{-1} \mathrm{~mol}^{-1}\right)\end{array}$} & \multirow{2}{*}{$\begin{array}{c}\Delta G^{\#} \\
\left(\mathrm{~kJ} \mathrm{~mol}{ }^{-1}\right)\end{array}$} \\
\hline & $298 \mathrm{~K}$ & $308 \mathrm{~K}$ & $318 \mathrm{~K}$ & & & \\
\hline$p-\mathrm{OCH}_{3}$ & 8.12 & 15.63 & 29.23 & 47.29 & -93.03 & 287.00 \\
\hline$p-\mathrm{CH}_{3}$ & 4.06 & 7.82 & 14.61 & 40.97 & -98.97 & 305.24 \\
\hline$p-\mathrm{C}_{6} \mathrm{H}_{5}$ & 2.96 & 5.71 & 10.67 & 38.68 & -101.08 & 311.71 \\
\hline$-\mathrm{H}$ & 2.67 & 5.14 & 9.61 & 36.19 & -102.42 & 315.81 \\
\hline$p-\mathrm{Cl}$ & 0.82 & 1.59 & 2.97 & 25.27 & -111.99 & 345.18 \\
\hline$p-\mathrm{Br}$ & 0.42 & 0.81 & 1.51 & 21.06 & -117.54 & 362.23 \\
\hline$m-\mathrm{NO}_{2}$ & 0.21 & 0.40 & 0.75 & 13.59 & -123.48 & 380.45 \\
\hline
\end{tabular}

${ }^{\mathrm{a}} 10^{3}[\text { Perborate }]_{0}=1.0 \mathrm{~mol} \mathrm{dm}^{-3} ; 10^{2}[5 \text {-oxoacid }]_{0}=4.0 \mathrm{~mol} \mathrm{dm}^{-3} ; 10^{2}\left[\mathrm{H}^{+}\right]=9.88 \mathrm{~mol} \mathrm{dm}^{-3} ;{ }^{*} 10^{4}[\mathrm{Ru}(\mathrm{III})]=1.0 \mathrm{~mol} \mathrm{dm}^{-3} ; \mathrm{HOAc}-\mathrm{H}_{2} \mathrm{O}=1: 1 \%(\mathrm{v} / \mathrm{v})$.

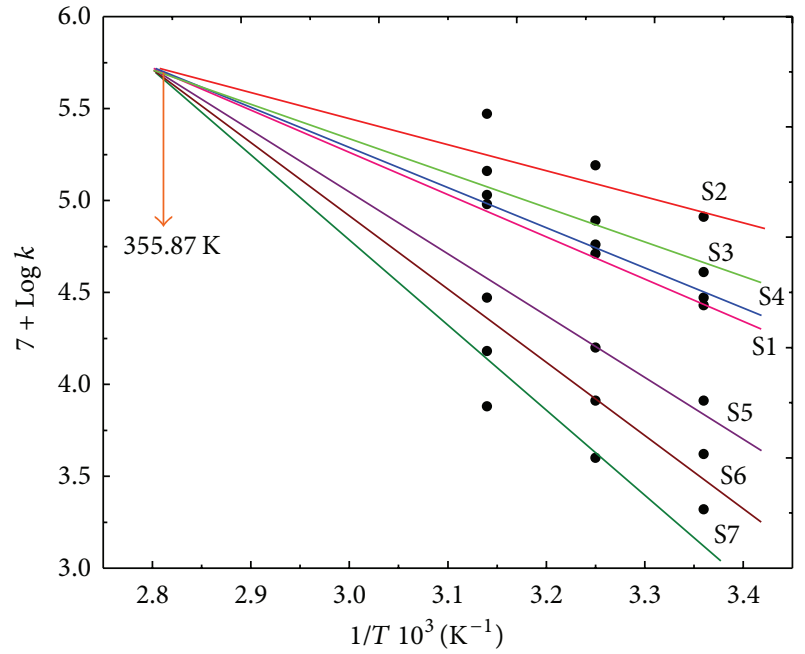

FIGURE 4: Arrhenius plots between $\log k$ and $1 / T$ showing the isokinetic temperature under the conditions of Table 9. (S1) $-\mathrm{H},(\mathrm{S} 2)$ $4^{\prime}$-methoxy, (S3) $4^{\prime}$-methyl, (S4) $4^{\prime}$-phenyl, (S5) $4^{\prime}$-chloro, (S6) $4^{\prime}$ bromo, (S7) $3^{\prime}$-nitro.

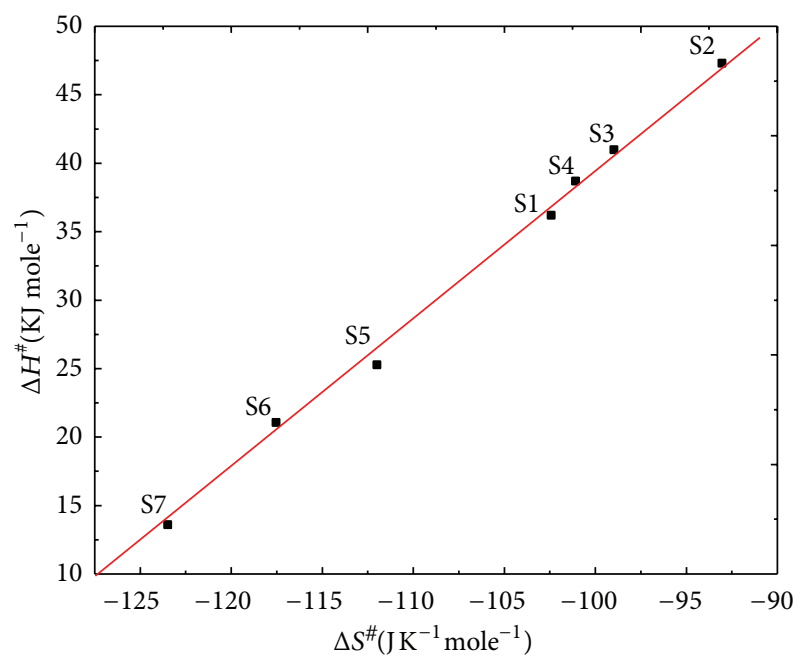

FIgURE 5: Plot between $\Delta H^{\#}$ and $\Delta S^{\#}$ : isokinetic relationship under the conditions of Table 9. (S1) - H, (S2) $4^{\prime}$-methoxy, (S3) $4^{\prime}$-methyl, (S4) $4^{\prime}$-phenyl, (S5) $4^{\prime}$-chloro, (S6) $4^{\prime}$-bromo, (S7) 3' -nitro.

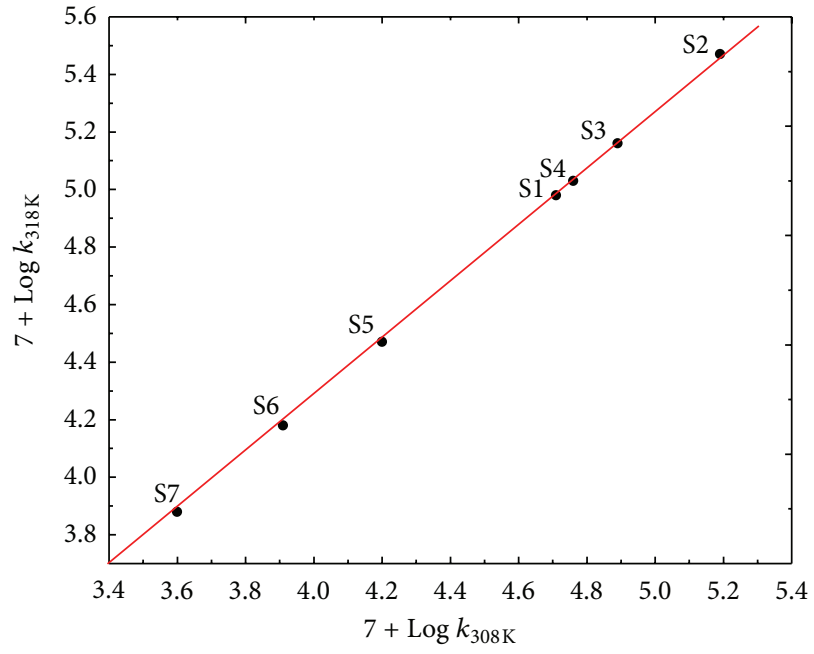

Figure 6: Exner's plot of $\log k_{318 \mathrm{~K}}$ against $\log k_{308 \mathrm{~K}}$ under the conditions of Table 9. (S1) -H, (S2) $4^{\prime}$-methoxy, (S3) $4^{\prime}$-methyl, (S4) $4^{\prime}$-phenyl, (S5) $4^{\prime}$-chloro, (S6) $4^{\prime}$-bromo, (S7) $3^{\prime}$-nitro.

The kinetic constants, calculated from the slope and intercept of the Hanes plot, are

$$
\begin{gathered}
K_{1} k_{3}=98.1 \mathrm{dm}^{3} \mathrm{~mol}^{-1} \mathrm{~s}^{-1}, \\
K_{2}=26.5 \mathrm{dm}^{3} \mathrm{~mol}^{-1}(\text { at } 308 \mathrm{~K}) .
\end{gathered}
$$

The rate law is compatible with the experimental results, namely, first order with respect to [perborate], zero order in $\left[\mathrm{H}^{+}\right]$, the Michaelis-Menten type dependence on [5oxoacid], almost the same rates of oxidation by perborate and hydrogen peroxide under identical conditions, the absence of enhancement or inhibition of perborate, hydrogen peroxide oxidations by borate or boric acid, and so forth.

\section{Conclusions}

Ruthenium(III) catalyzed perborate oxidation of substituted 5-oxoacids in acidic solution is associated with isokinetic relationship. In aqueous solution, perborate generates hydrogen peroxide. Under the conditions $[\mathrm{Ru}(\mathrm{III})] \lll$ 


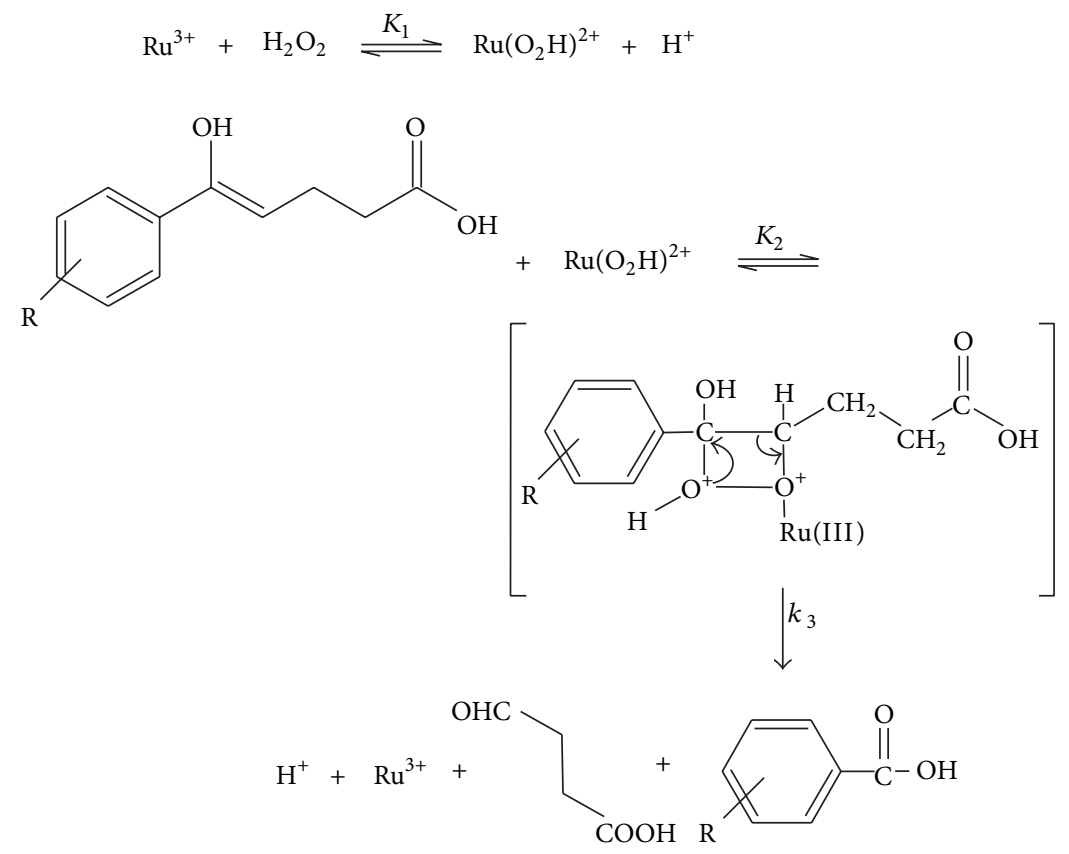

(6)

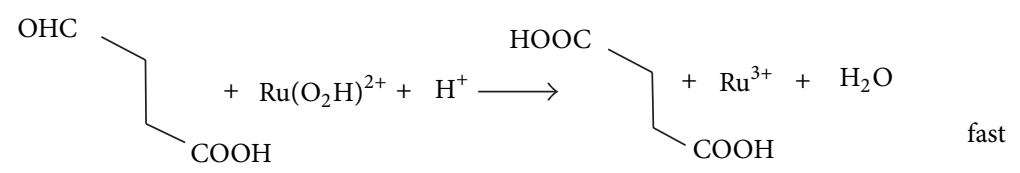

SCHEME 4

[perborate $]_{0} \ll[5 \text {-oxoacid }]_{0}$, the oxidation is first order with respect to the oxidant and catalyst. The rate of catalyzed oxidation displays the Michaelis-Menten kinetics on the reductant and is independent of $\left[\mathrm{H}^{+}\right]$of the medium. Hydrogen peroxide is the reactive species of perborate, and the kinetic results reveal formation of ruthenium(III) peroxo species-5-oxoacid complex. The variation of oxidation rate with ionic strength of the medium is quite small at low $\left[\mathrm{H}^{+}\right]$ but appreciable at high $\left[\mathrm{H}^{+}\right]$. The oxidation rate increases with decreasing dielectric constant of the medium at low and high acidities. The rates of ruthenium(III) catalyzed perborate and hydrogen peroxide oxidations are almost the same. Borate and boric acid do not influence the oxidations. Electron-releasing substituents accelerate the reaction rate and electron-withdrawing substituents retard the reaction. The order of reactivity among the studied 5-oxoacids is $p$ methoxy $\gg p$-methyl $>p$-phenyl $>-\mathrm{H}>p$-chloro $>p$ bromo $>m$-nitro. Activation parameters have been evaluated using Arrhenius and Eyring's plots. A mechanism consistent with the observed kinetic data is proposed and discussed. A suitable rate law is derived based on the mechanism. The experimental protocol suggests that this reaction could find utility as a regioselective route for the synthesis of carboxylic acids, specially substituted benzoic acids.

\section{Conflict of Interests}

The authors declare that there is no conflict of interests regarding the publication of this paper.

\section{References}

[1] F. A. Cotton and G. Wilkinson, Advanced Inorganic Chemistry, Wiley Interscience, New York, NY, USA, 5th edition, 1988.

[2] T. M. Connor and R. E. Richards, "Nuclear resonance spectra of some peroxy-salts," Journal of the Chemical Society, pp. 289-293, 1958.

[3] J. O. Edwards and V. F. Ross, "The structural chemistry of the borates," in The Chemistry of Boron and Its Compounds, E. L. Muetterties, Ed., p. 192, John Wiley and Sons, New York, NY, USA, 1967.

[4] A. Mckillop and W. R. Sanderson, "Sodium perborate and sodium percarbonate: further applications in organic synthesis," Journal of the Chemical Society, Perkin Transactions, vol. 1, pp. 471-479, 2000.

[5] C. Karunakaran and B. Muthukumaran, "Molybdenum(VI) catalysis of perborate or hydrogen peroxide oxidation of iodide ion," Transition Metal Chemistry, vol. 20, no. 5, pp. 460-462, 1995.

[6] C. Karunakaran and R. Kamalam, "On the mechanism of the perborate oxidation of organic sulfides in glacial acetic acid," European Journal of Organic Chemistry, no. 19, pp. 3261-3263, 2000.

[7] C. Karunakaran and P. Manimekalai, "Kinetics and mechanism of perborate oxidation of organic sulphides," Tetrahedron, vol. 47, no. 41, pp. 8733-8738, 1991.

[8] C. Karunakaran and R. Kamalam, "Mechanism and reactivity in perborate oxidation of anilines in acetic acid," Journal of the Chemical Society. Perkin Transactions 2, no. 12, pp. 2011-2018, 2002. 
[9] D. Kungumathilagam and K. Karunakaran, "Kinetics and mechanism of meso-tetraphenylporphyriniron(III) chloride (TPP) catalysed oxidation of indole by sodium perborate," Polish Journal of Chemical Technology, vol. 15, no. 2, pp. 107-111, 2013.

[10] S. Shree Devi, B. Muthukumaran, and P. Krishnamoorthy, "Vanadium $(\mathrm{V})$ catalysis of perborate oxidation of substituted 5oxo acids: a kinetic and mechanistic study," Ionics, 2014.

[11] N. P. Marigangaiah and K. K. Banerji, "Kinetics \& mechanism of oxidation of acetone, butanone, pentan-2-one, 3-methylbutan2-one, hexan-2-one \& 4-methylpentan-2-one by acid permanganate," Indian Journal of Chemistry A, vol. 14, pp. 660-662, 1976.

[12] A. Meenakshi and M. Santhappa, "Oxidation studies involving Tl(III)," Indian Journal of Chemistry, vol. 11, pp. 393-394, 1973.

[13] N. A. M. Farook, "Kinetics of oxidation of 4-oxoacids by Nchlorosaccharin in aqueous acetic acid medium," Journal of Solution Chemistry, vol. 36, no. 3, pp. 345-356, 2007.

[14] D. F. Gnana Rani, F. J. Maria Pushparaj, I. Alphonse, and K. S. Rangappa, "Kinetics and mechanism of oxidation of 4-oxoacids by hexacyanoferrate(III) catalysed by Os(VIII)," Indian Journal of Chemistry B, vol. 41, no. 10, pp. 2153-2159, 2002.

[15] K. H. Stern and A. E. Martell, "Thermodynamics of ion pair formation in some non-aqueous solvents," Journal of the American Chemical Society, vol. 77, no. 7, pp. 1983-1986, 1955.

[16] A. I. Vogel, Text Book of Quantitative Chemical Analysis, ELBS Longman, New York, NY, USA, 5th edition, 1958.

[17] R. N. Haward, "The fast and slow extension of some plastic materials," Transactions of the Faraday Society, vol. 39, pp. 267280, 1943

[18] F. R. Duke, “The disproportionation of manganate ion. Managanese dioxide as heterogeneous catalyst," The Journal of Physical Chemistry B, vol. 56, no. 7, pp. 882-884, 1952.

[19] M. G. Alder and J. E. Leffler, "The role of the solvent in radical decomposition reactions: phenylazotriphenylmethane," Journal of the American Chemical Society, vol. 76, no. 5, pp. 1425-1427, 1954.

[20] F. Feigl and V. Anger, Spot Tests in Organic Analysis, Elsevier, Amsterdam, The Netherlands, 1975.

[21] J. E. Leffler and E. Grunwald, Rates and Equilibrium of Organic Reactions, Wiley, New York, NY, USA, 1963.

[22] G. Sikkandar, K. A. B. Ahamed, and S. Kannan, "Kinetics of oxidation of 4-oxoacids by permanganate in buffer media," Indian Journal of Chemistry A, vol. 38, no. 2, pp. 183-186, 1999.

[23] C. J. Adams and I. E. Clark, "On the nature of the peroxoborate ion in solution," Polyhedron, vol. 2, no. 7, pp. 673-675, 1983.

[24] R. Pizer and C. Tihal, "Peroxoborates. Interaction of boric acid and hydrogen peroxide in aqueous solution," Inorganic Chemistry, vol. 26, no. 21, pp. 3639-3642, 1987.

[25] G. M. Panchenkov and V. P. Lebedev, Chemical Kinetics and Catalysis, Mir, Moscow, Russia, 1976.

[26] J. A. Connor, E. A. V. Ebsworth, H. J. Emeleus, and A. G. Sharpe, Eds., Advances in Inorganic Chemistry and Radiochemistry, Academic, New York, NY, USA, 1964.

[27] D. C. Bradley and P. Thornton, Comprehensive Inorganic Chemistry, vol. 3, Pergamon, Oxford, UK, 1973, edited by J. C. Bailar, Jr., H. J. Emeleus, R. Nyholm. 

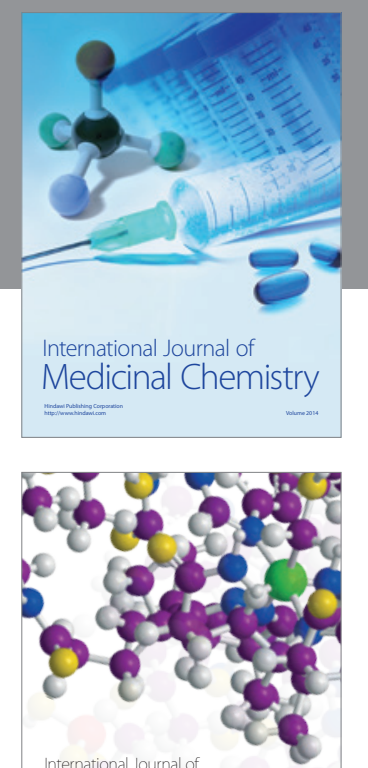

\section{Carbohydrate} Chemistry

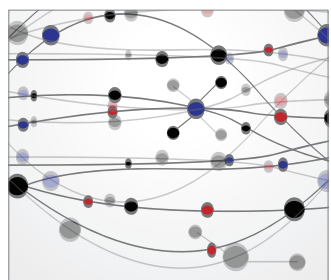

The Scientific World Journal
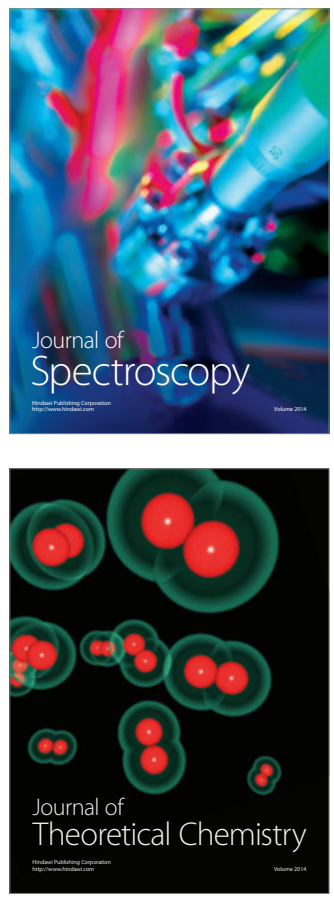
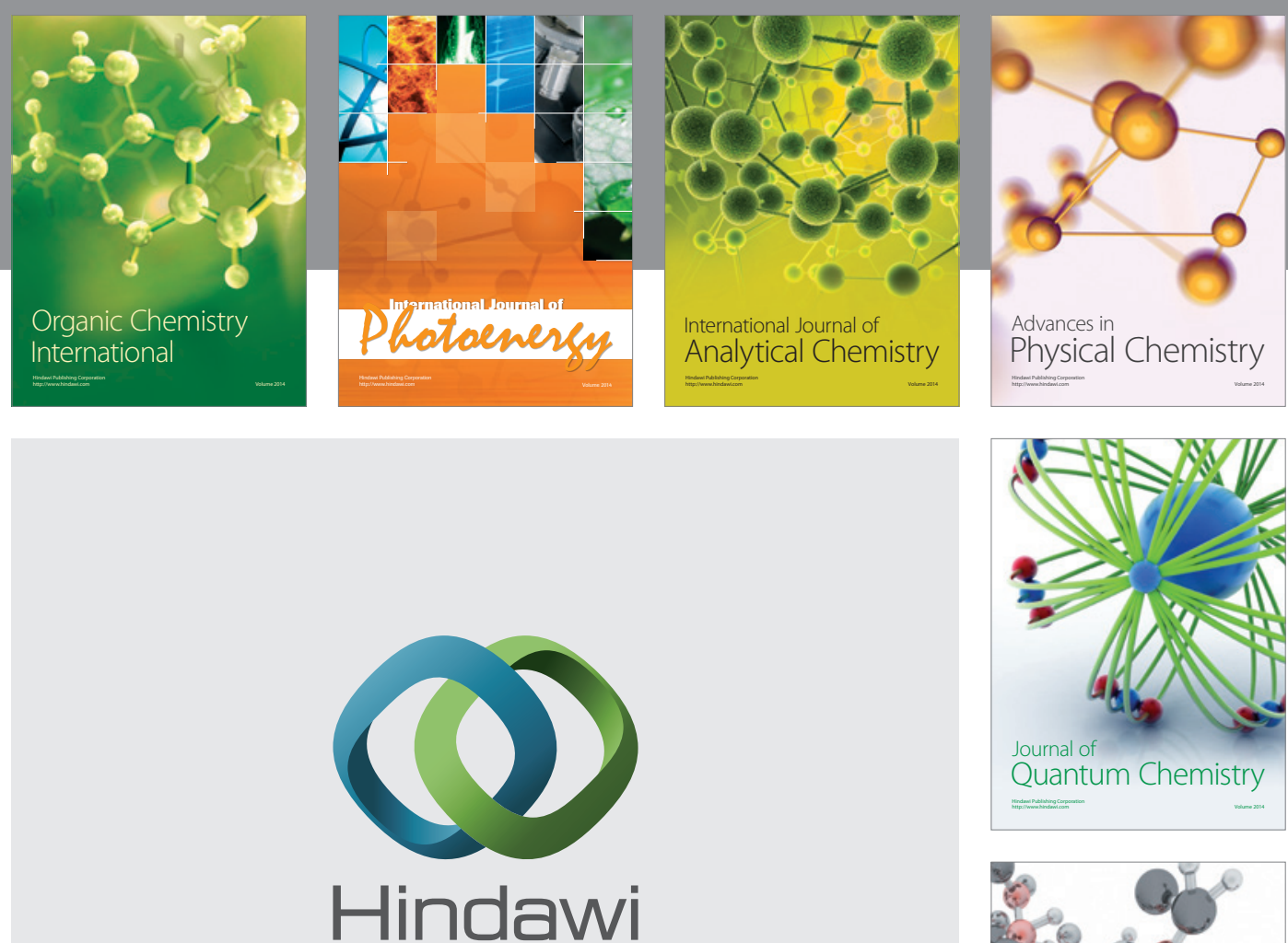

Submit your manuscripts at

http://www.hindawi.com

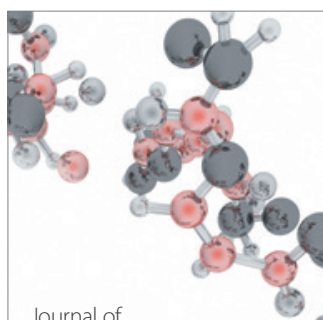

Analytical Methods

in Chemistry

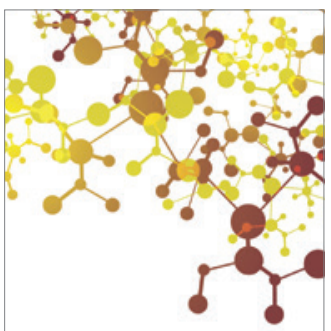

Journal of

Applied Chemistry

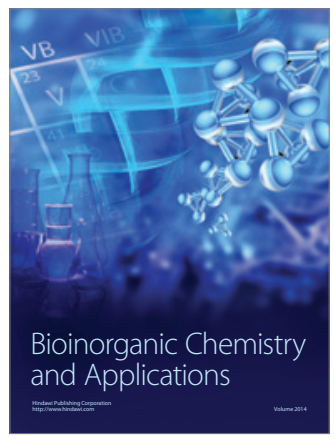

Inorganic Chemistry
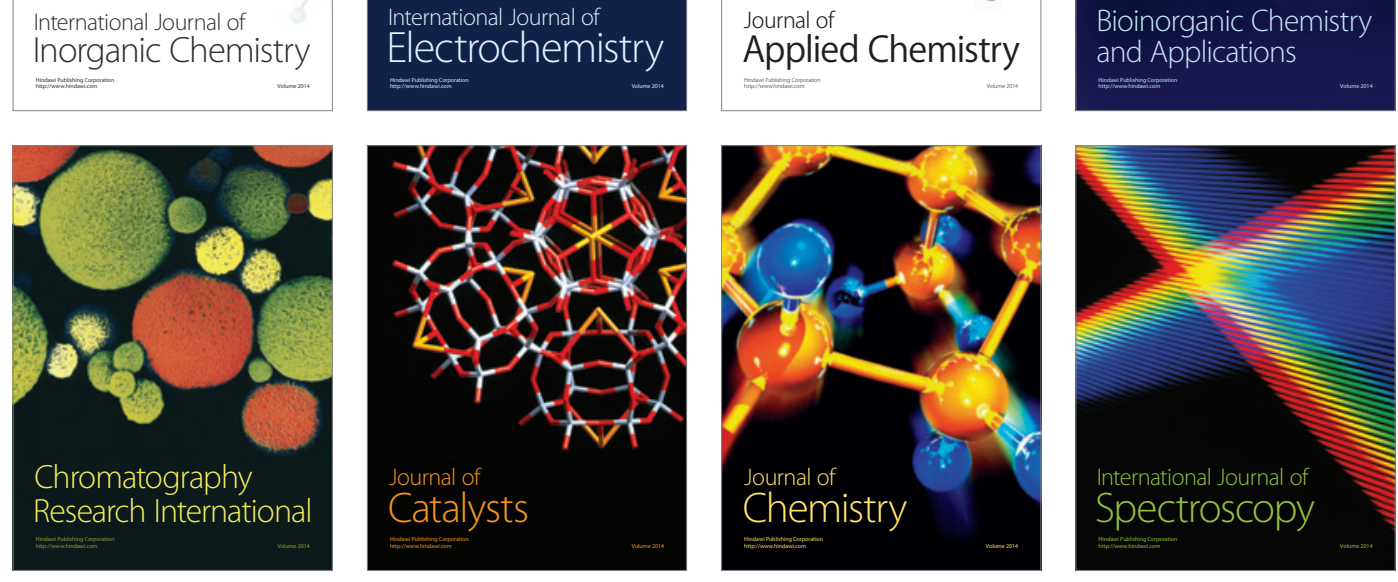